\title{
Simulation of Chassis Motion of Harvester Anti - rollover
}

\section{Song Xiangwen ${ }^{1, a}$, Cao Shukun²※,b(Corresponding author), Li Shizhuang ${ }^{3, c}$, Shen $\mathrm{HaO}^{4, \mathrm{~d}}$}
${ }^{1}$ Nanxinzhuang West Road 336Jinnan, School of Mechanical Engineering, University of Jinan, Jinan 250022, Shandong Province, China
${ }^{2}$ Nanxinzhuang West Road 336Jinnan, School of Mechanical Engineering, University of Jinan, Jinan 250022, Shandong Province, China
${ }^{3}$ Nanxinzhuang West Road 336Jinnan, School of Mechanical Engineering, University of Jinan, Jinan 250022, Shandong Province, China
${ }^{4}$ Nanxinzhuang West Road 336Jinnan, School of Mechanical Engineering, University of Jinan, Jinan 250022, Shandong Province, China
a751937831@qq.com b87756997@qq.com c1148660320@qq.com d354174809@qq.com

Key words: anti-rollover; Corn harvester; oil and gas suspension; Motion simulation

Abstract: Based on the oil and gas suspension of the harvester chassis, in the CAD simulation of the cylinder movement to the maximum limit position, the frame deflection, and ultimately determine the limit position, the hydraulic cylinder and thrust lever offset angle to see whether the hydraulic cylinder and thrust The maximum permissible angle of the rod within the range, to prevent the movement of hydraulic cylinders and thrust rods interfere with each other, and pull off the thrust rod or damage the connection seat.

\section{Introduction}

As the oil and gas suspension in the adjustment of the body balance process, due to the harvester installed up and down, left and right offset, resulting in hydraulic cylinder and thrust bar before and after the left and right offset. When the hydraulic cylinder and thrust rod offset beyond

$2 \%$. Corresponding author:

Cao Shukun(1970-), male(Han nationality),School of Mechanical Engineering. University of Jinan,Ph.D,M ainly engaged in mechanical CAD / CAE / CAM , NC technology, agricultural machinery and equipment, and other aspects of the study.Email: 87756997@qq.com; Telephone:18769716867

About the Author:

1、Song Xiangwen (1993-), male(Han nationality),School of M echanical Engineering. University of Jinan,Postgraduate, M ainly engaged in agricultural machinery.

3、 Li Shizhuang(1991-),male(Han nationality),School of Mechanical Engineering. University of Jinan,Postgraduate,Mainly engaged in grain drying research.

4、Shen Hao(1990-),male(Han nationality),School of Mechanical Engineering. University of Jinan,Postgraduate,Mainly engaged in agricultural machinery.

Fund Project:

1. Shandong Province, the major project of science and technology (item number: 2015ZDZX10001) " the development and industrialization demonstration of intelligent corn combine harvester" .

2、 Taishan industry leading talent project special funds. 
the hydraulic cylinder and thrust rod of the maximum deviation of the angle, the hydraulic cylinder and thrust bar damage or hydraulic cylinder and thrust rod movement interfere with each other, especially the thrust rod, when the hydraulic cylinder Leveling, because the thrust lever deflection angle exceeds the maximum deflection angle of the thrust rod, with the hydraulic cylinder telescopic, will pull the thrust bar, leaving the suspension can not work properly. Therefore, this chapter will discuss whether the maximum deflection angle of the hydraulic cylinder and the thrust rod is smaller than the maximum universal angle of the hydraulic cylinder and the thrust rod when the hydraulic cylinder is adjusted to the limit position. As the harvester in the uphill, downhill, walking slope, in the leveling, the axle tilt, adjust the body to the level, to see its maximum leveling range. But because the axle tilt when the chassis movement simulation and axle level to adjust the body tilt to find the body adjustment angle, hydraulic cylinder deflection angle, thrust bar deflection angle, and the axle level, the movement simulation more intuitive, so this article uses the car Bridge level, adjust the body to the maximum deflection angle, measuring the various angles.

\section{Before and after leveling, the hydraulic cylinder and thrust bar deflection angle}

When the harvester climbed or downhill, due to serious tilt before and after, especially when the harvester downhill, due to the front of the harvester center, it is easy to cause the front turn. As the front and rear adjustment caused by the hydraulic cylinder and thrust bar deflection, so this article only discuss the front side of the increase, the rear side of the situation. As the front and rear leveling, longitudinal thrust rods can be moved according to the frame rotation, lateral offset less, and horizontal thrust bar with the frame up and down at the same time, because the overall frame backward, the horizontal thrust lever will The direction of longitudinal deviation before and after the occurrence of here, we have to discuss the horizontal thrust lever longitudinal offset angle is less than the thrust rod of the universal deflection angle.

In this case, when the suspension cylinder is in the middle position as the starting level, we set the hydraulic cylinder stroke to plus or minus $100 \mathrm{~mm}$, the front side hydraulic cylinder is elongated by $100 \mathrm{~mm}$, the rear hydraulic cylinder retracts $100 \mathrm{~mm}$ as the leveling limit Point, the whole body tilt angle of $3.58^{\circ}$.

Before the bridge hydraulic cylinder base for the center to $833+100$, that is, $933 \mathrm{~mm}$ for the radius of the circle, the same reason, after the bridge base for the center to $733 \mathrm{~mm}$ radius for the circle, and then make a tilt $3.58^{\circ}$, length of $3200 \mathrm{~mm}$ straight line, that And the front axle circle tangent, and the rear axle round tangent, then the slash and circle cut point is the rotation after the hydraulic cylinder and frame seat connection point. The frame, front and rear axle horizontal thrust bar and the intersection of the frame, the vertical thrust bar and the intersection of the frame made block, as a whole. The whole frame is rotated so that the center of the front and rear frames and the center of the hydraulic cylinder are overlapped at the front and rear points, respectively, Coincide with the rear frame is the position of the rear frame, before and after leveling, the frame rotation diagram shown in Fig1. 


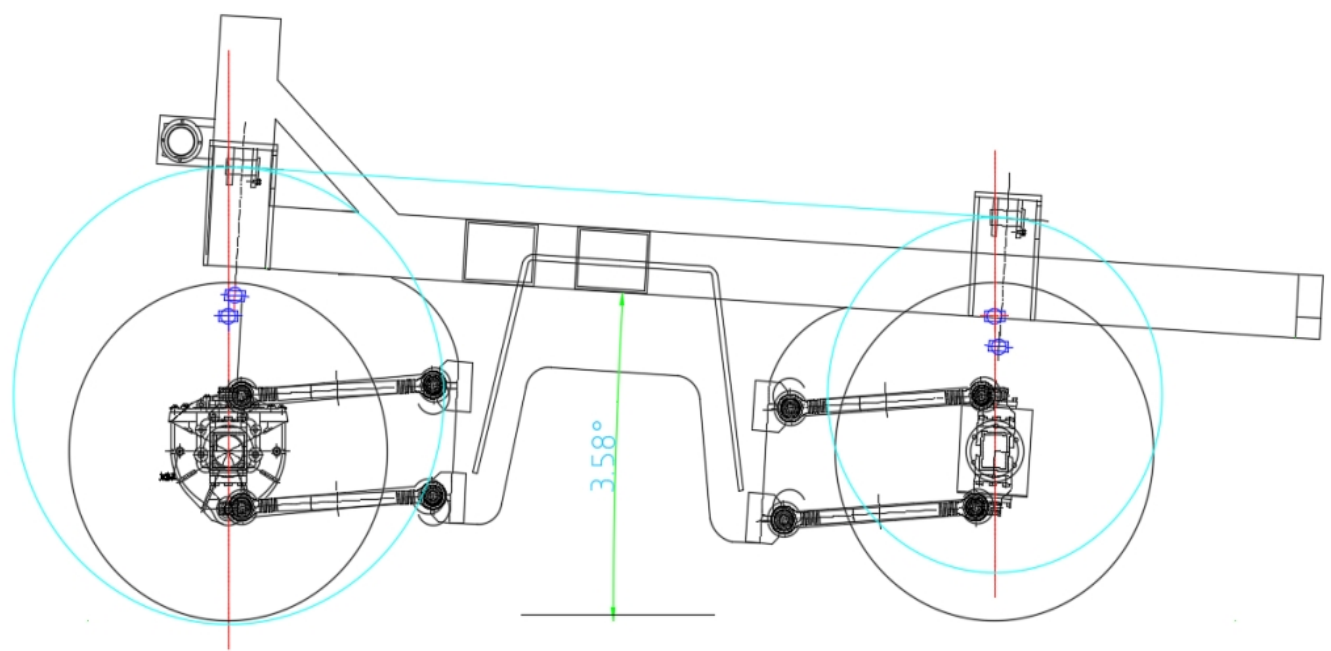

Fig 1. Adjust the frame before and after leveling

At this point, the body before and after the deflection angle of $3.58^{\circ}$, hydraulic cylinder deflection angle of $4.28^{\circ}<13^{\circ}$, meet the conditions. At this time, the transverse thrust lever is shifted by $26.8 \mathrm{~mm}$ in the longitudinal direction, and the longitudinal displacement angle of the lateral thrust lever is $\alpha=\arctan \frac{H}{L}=\arctan \frac{26.8}{750}=2.05^{\circ}$ While the deflection angle of $3.59^{\circ}$, and our thrust rod of the universal deflection angle of $6.5^{\circ}$ side, meet the conditions. The deflection position of the thrust rod connector is shown in Fig 2 below.

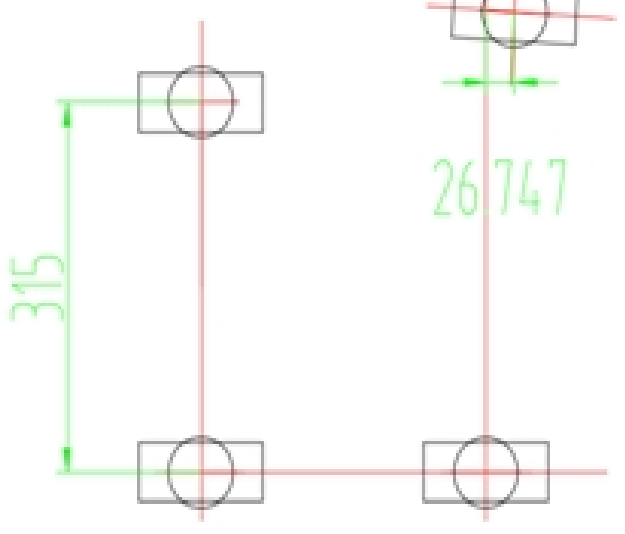

Fig2 .Flattened end point, thrust lever connector deflection position diagram

\section{Around the leveling, the hydraulic cylinder and thrust bar deflection angle}

\section{Frame left high and low, body leveling}

When the harvester is sloping or turning, the harvester is deflected due to the center of the harvester. This requires the harvester to be horizontally flattened. When the suspension is horizontal leveling, because the lateral thrust bar with the frame rotation, very few longitudinal offset, but the vertical thrust bar in addition to the body up and down in addition to the rotation, but also horizontal offset, in the left and right leveling, We will discuss whether the longitudinal thrust lever lateral angle is less than the universal deflection angle of the thrust rod.

When the right side of the hydraulic cylinder retracted $100 \mathrm{~mm}$, the left side of the hydraulic 
cylinder elongation of $100 \mathrm{~mm}$, analysis of longitudinal thrust lever lateral offset. After the bridge as an example, to the right side of the center of the axle as the center of the center, to $833 \mathrm{~mm}-100 \mathrm{~mm}$ radius of the circle to the left side of the axle base to $833 \mathrm{~mm}+100 \mathrm{~mm}$ radius of the circle, while the horizontal thrust lever in the axle The upper base for the center of the horizontal thrust rod length of $750 \mathrm{~mm}$ for the radius of the circle. At the same time connect the left ear center of the frame, the center of the right ear, the horizontal thrust rod connecting seat, draw the triangle, turn the triangle, so that the center of the horizontal thrust rod is rotated in the transverse thrust bar circle, , While the time to meet the position for the frame rotation, The rotation diagram is shown in Fig 3 below.

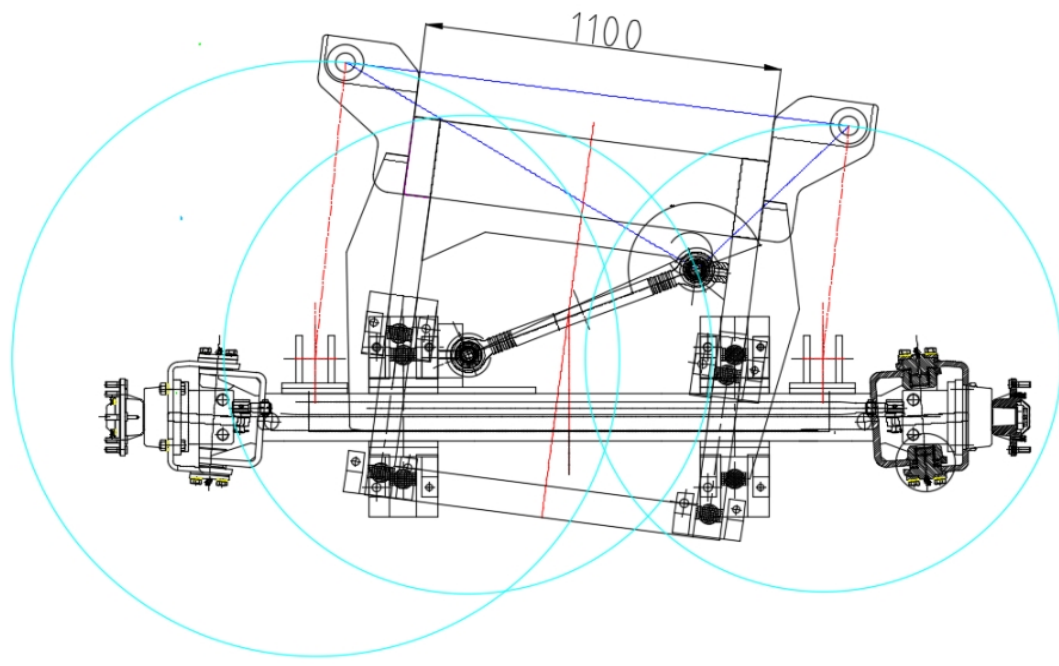

Fig 3 Left, right, and frame

At this time deflection angle as shown in Figure 3-6, body deflection $7.35^{\circ}$, while the hydraulic cylinder deflection $7^{\circ}<13^{\circ}$, meet the conditions. The lower right side of the thrust lever of the largest displacement, displacement as shown in Fig4: longitudinal thrust bar lateral offset $77.59 \mathrm{~mm}, \quad \alpha=\arctan \frac{\mathrm{H}}{\mathrm{L}}=\arctan \frac{77.59}{800}=5.5^{\circ}<6.5^{\circ}, \quad$ Meet the thrust bar deflection angle requirements.
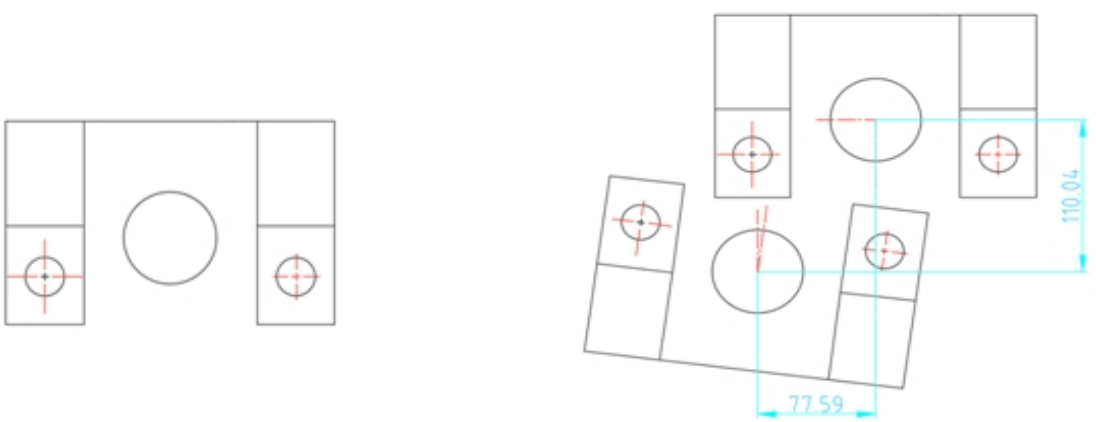

(a) The joint is overlapped in the stationary state (b) The position of the joint in the leveling state Fig 4 Left and right when the vertical thrust lever to move the position

\section{When the frame is right high and low, the body is flat}

As the horizontal thrust bar is not symmetrical, so about leveling, the two cases should be 
considered. When the right side of the elongation of $100 \mathrm{~mm}$, the left side of the reduction of $100 \mathrm{~mm}$, leveling motion mapping method and the same high left and right low. The schematic diagram of the rotation structure of the right extension left frame is shown in Figure 5 below.

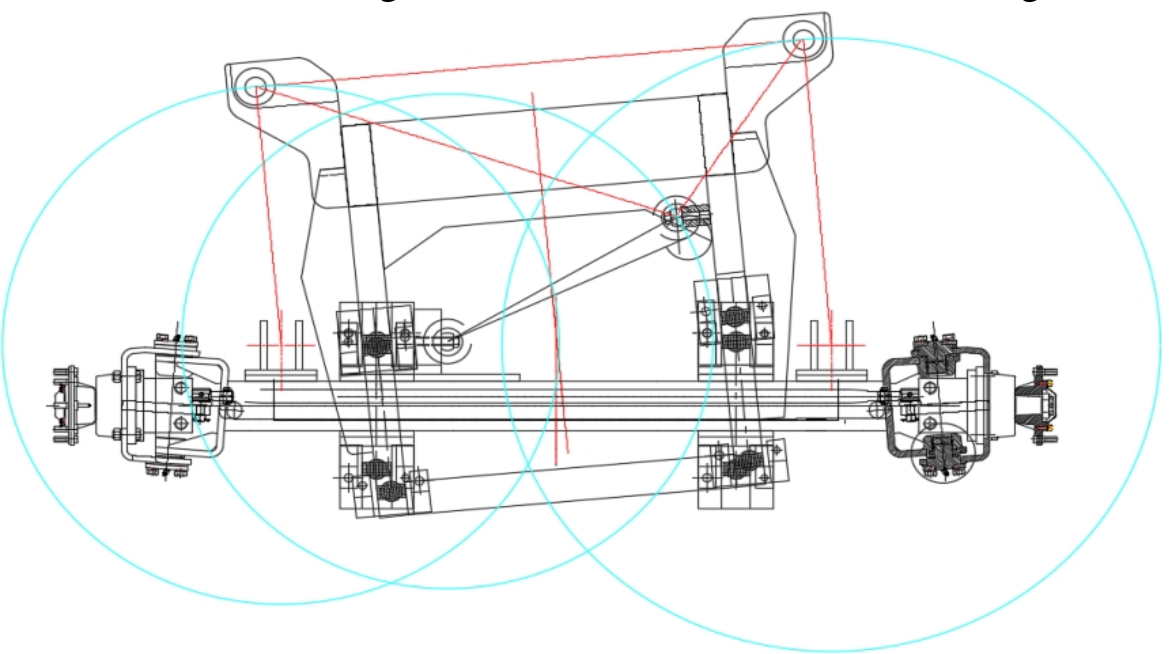

Figure 5 Schematic diagram of the rotation of the right frame

The end of rotation, body deflection angle of $5.27^{\circ}$, hydraulic deflection angle of $5.21^{\circ}<13^{\circ}$, meet the conditions. Vertical thrust bar lateral displacement As shown in Figure 6, the vertical thrust lever lateral offset $\alpha=\arctan \frac{\mathrm{H}}{\mathrm{L}}=\arctan \frac{41.38}{800}=2.98^{\circ}<6.5^{\circ}$, meet the thrust bar deflection angle requirements.

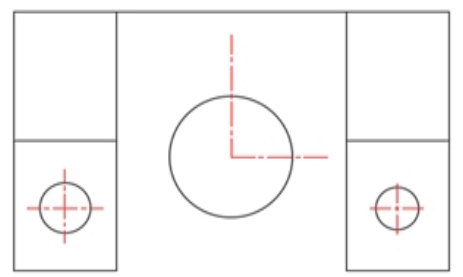

(a) The stationary state connector is overlapped

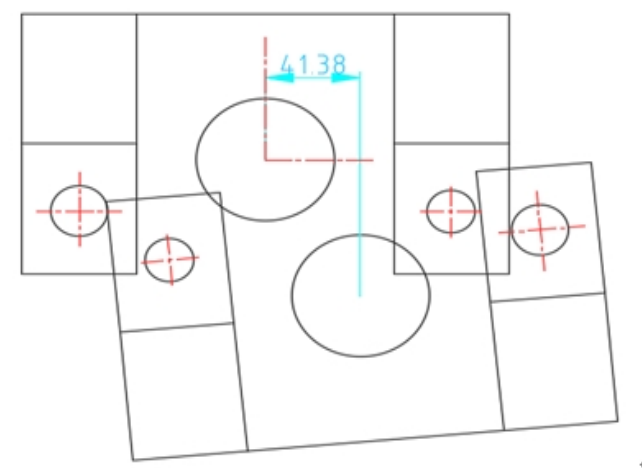

(b) The position of the connector in the leveling state Figure 6 Vertical thrust lever lateral offset diagram

\section{Conclusions}

This chapter determines whether the deflection angle of the hydraulic cylinder and the thrust rod is in accordance with the movement of the hydraulic cylinder and the horizontal and longitudinal thrust rods by simulating the leveling of the front and rear of the frame, Hydraulic cylinder and thrust rod of the largest universal angle to prevent the deflection is too large and cause the thrust bar was pulled off, and can not work properly.

\section{Acknowledgements}

This work was financially supported by the Shandong Province, the major project of science and technology (item number: 2015ZDZX10001) " the development and industrialization demonstration of intelligent corn combine harvester" .and Taishan industry leading talent project 
special funds.

\section{References}

[1] Pevsner J.M.,EquaizingtyPesofsusPension,AutomobileEngineer,Jan.1957:10.16P.

[2] Geoff Rideout,Dynamic testing and modeling of the interconnected moulton hydragas suspension system,Master of Science dissertation,Queen's University at Kingston,1999.

[3] Cao D, Rakheja S, Su C Y. Roll plane analysis of interconnected hydro-pneumatic suspension struts[C]//ASME 2005 International Mechanical Engineering Congress and Exposition. American Society of Mechanical Engineers, 2005: 133-142.

[4] Bauer Wolfgang, Hydropneumatic suspension system[M]. Verlag Berlin Heidelberg: springer,2011.

[5] H.G.Gibson,K.C.Elliott. Side slope stability of articulated-frame logging tractors[J].Journal of Terra mechanics, 1971(2): 65-79. 